\title{
Bioisosteres in drug discovery: focus on tetrazole
}

\author{
Yulin Zou*,1, Li Liư ${ }^{1}$, Junxiong Liu ${ }^{1}$ \& Guocheng Liu ${ }^{1}$ \\ ${ }^{1}$ The Third Clinical Medical College of the Three Gorges University, Gezhouba Central Hospital of Sinopharm, Yichang 443002, \\ Hubei, PR China \\ *Author for correspondence: zouyulin1213@163.com
}

"Tetrazole, which is usually used as the bioisoster of carboxylic acid, is metabolically stable to many of the biological transformations that the functionality of carboxylic acid is susceptible to in the liver"

First draft submitted: 16 October 2019; Accepted for publication: 18 October 2019; Published online: 25 November 2019

Keywords: antibacterial • anticancer • antifungal • antihypertensive $\bullet$ tetrazole

\section{Basic structure \& significance of tetrazole}

Tetrazole, a doubly unsaturated five-membered ring containing four nitrogen atoms and one carbon atom, can serve as precursors for a variety of nitrogen-containing heterocycles by the Huisgen rearrangement [1]. Tetrazole, which is usually used as the bioisoster of carboxylic acid, is metabolically stable to many of the biological transformations that the functionality of carboxylic acid is susceptible to in the liver [2]. Some prodrug approaches to tetrazole drugs have been implemented to improve oral bioavailability and replacement of carboxylic acid by tetrazole has the potential to increase the lipophilicity and bioavailability and reduce the side effects of drugs [3]. Moreover, tetrazole has the capacity to exert various noncovalent interactions with biological targets and its derivatives possess diverse pharmacological properties, such as anti-Alzheimer's disease [4], antiangiogenic [5], antibacterial [6], anticancer [7], antifungal [8], antimalarial [9], antitubercular [10] and antiviral [11] activities. Notably, the tetrazole-based drugs such as cefamandole, ceftezole, losartan and valsartan have already been used in clinics for the treatment of various diseases and tetrazole derivatives as pharmacologically significant scaffolds have been of great interest in recent years.

\section{Tetrazoles as antibacterial agents}

Bacterial infections are responsible for a vast majority of infections such as pneumonia, wound infections, bloodstream infections and sexually transmitted diseases in hospital and community settings and they remain one of the main causes of morbidity and mortality throughout the world [12]. Antibiotics are commonly used to fight against bacterial infections and some clinically used antibacterial agents contain tetrazole skeleton, revealing the potential of tetrazole derivatives as putative antibacterial drugs.

Ceftezole (the first-generation cephalosporin antibiotic) and cefamandole (known as cephamandole, a secondgeneration cephalosporin antibiotic) bearing a tetrazole moiety could inhibit bacterial cell wall synthesis and possess broad-spectrum antibacterial activity against both Gram-positive and Gram-negative organisms even in drug-resistant pathogens. Tedizolid (second-generation oxazolidinone), an oxazolidinone-class antibiotic prodrug, has already been approved for the treatment of acute bacterial skin and skin structure infections caused by susceptible isolates of several Gram-positive bacteria. The antibacterial activity of tedizolid is mediated by binding to the $50 \mathrm{~S}$ subunit of the bacterial ribosome, resulting in the inhibition of protein synthesis. The mechanism of action is different from that of other nonoxazolidinone-class antibacterial drugs, so tedizolid is also sensitive to some drugresistant pathogens. For example, tedizolid is 4- to 16-fold more active than linezolid against methicillin-resistant Staphylococcus aureus (MRSA). DA-7867, the amide analog of tedizolid, also exhibited promising activities against a panel of clinically important bacteria including drug-resistant pathogens. The above facts demonstrated the possible utility of the tetrazole scaffolds in the development of new antibacterial agents [6]. 


\section{Tetrazoles as potential anticancer agents}

The incidence of cancers leads to 9 million related deaths annually and around 100 million people will die in the next 10 years if the trends continue; cancers have already put a tremendous burden on the global health system [13].

The tetrazole derivative letrozole, has already been used in clinics to treat certain types of breast cancer, usually after resection and failure of tamoxifen. Letrozole, a nonsteroidal aromatase inhibitor, prevents aromatase from producing estrogens by competitive, reversible binding to the heme of its cytochrome P450 unit. Encequidar (HM30181) could effectively block transepithelial transport of paclitaxel in MDCK monolayers and its inhibitory activity toward P-glycoprotein (P-gp) was 20-50-times higher than that of tariquitar. Encequidar as a thirdgeneration P-gp inhibitor could increase the oral bioavailability of co-administered paclitaxel by more than 12 times in rats and is currently under development to enhance the oral absorption of P-gp substrates [7].

\section{Tetrazoles as potential antifungal agents}

The life-threatening invasive fungal infections are as a major threat in critically ill adult and pediatric patients, leading to over 1.35 million deaths each year globally [14]. The side effects caused by the antifungal agents and the emergence of drug-resistant fungi could result in the complication of treatment of fungal infections, calling for the development of novel antifungal agents.

Oteseconazole (VT-1161), a tetrazole-pyridine hybrid, is a fungal-specific cytochrome P51 inhibitor with greater selectivity, fewer side effects and improved potency than current treatment options. Oteseconazole, a potential best-in-class treatment option for patients suffering from recurrent vulvovaginal candidiasis, is the first US FDA approved drug for the treatment of this common disease. Besides which, oteseconazole has strong potential in the fight against other fungal infections, including azole-resistant fungal infections [15]. Quilseconazole (VT-1129), a derivative of oteseconazole, is also a fungal-specific cytochrome P51 inhibitor. Quilseconazole, as a promising oral agent, has the potential to fight against cryptococcal meningitis, a life-threatening fungal infection of the brain and the spinal cord that occurs most frequently in patients with HIV infection, transplant recipients and oncology patients. Quilseconazole has received fast track, orphan drug designation and is considered a qualified infectious disease product by the FDA for the treatment of cryptococcal meningitis.

\section{Tetrazoles as antihypertensive agents}

Hypertension, also known as high blood pressure, contributes to the burden of heart disease, stroke and kidney failure and premature mortality and disability. Hypertension accounts for at least $45 \%$ of deaths due to heart disease and $51 \%$ of deaths due to stroke, leading to 9.4 million deaths worldwide every year [16].

Some tetrazole-containing drugs such as losartan, valsartan, irbesartan and candesartan have already been used for the treatment of hypertension in clinical practice, demonstrating the potential of tetrazole derivatives as putative antihypertensive drugs [17]. Losartan, as the first angiotensin-II receptor antagonist, can keep blood vessels from narrowing, which lowers blood pressure and improves blood flow. Moreover, it could also be used in the management of hypertension and heart failure, particularly in patients who develop cough with angiotensin-converting enzyme inhibitors. Valsartan, irbesartan and candesartan also belong to angiotensin receptor blockers and they can relax the blood vessels and put less strain on the heart via blocking the action of certain natural substances that tighten the blood vessels. Valsartan is used as an oral medication to treat hypertension, heart failure and diabetic kidney disease in adults and children older than 6 years of age. Irbesartan is used to treat hypertension and protect the kidneys from damage due to diabetes whereas candesartan is used in the treatment of hypertension and congestive heart failure.

\section{Tetrazoles with miscellaneous biological activity}

Besides the excellent antibacterial, anticancer, antifungal and antihypertensive potency, tetrazoles also showed promising in vitro and in vivo activity against Alzheimer's disease, asthma, malaria, tuberculosis and virus. Some of them not only exhibited great activity against drug-sensitive organisms, but also demonstrated potential in vitro and in vivo activity against drug-resistant, including multidrug-resistant and extensively drug-resistant pathogens. Moreover, several of them have already been used in clinical practice, such as tomelukast (LY171883), a leukotriene antagonist, and they also showed potential activity against Alzheimer's disease [18]. 


\section{Conclusion}

Tetrazole, the bioisoster of carboxylic acid, is an important pharmacophore in the development of novel drugs. Tens of tetrazole-containing compounds, such as cefamandole, ceftezole, tedizolid, letrozole, encequidar, oteseconazole, quilseconazole, losartan, valsartan, irbesartan, candesartan and tomelukast, have already been used in clinics for antibacterial, antiasthmatic, anticancer, antifungal and antihypertensive purposes, and diverse tetrazoles also endowed with promising in vitro and in vivo anti-Alzheimer's disease, antimalarial, antitubercular and antiviral pharmacological properties, demonstrating their potential for clinical deployment in the control and eradication of various diseases.

The structure-activity relationships of tetrazoles were also enriched by the recent studies and the enriched structure-activity relationships may set up the direction for the design and development of novel tetrazoles with broader spectrum, higher efficiency and lower toxicity.

\section{Financial \& competing interests disclosure}

The authors have no relevant affiliations or financial involvement with any organization or entity with a financial interest in or financial conflict with the subject matter or materials discussed in the manuscript. This includes employment, consultancies, honoraria, stock ownership or options, expert testimony, grants or patents received or pending, or royalties.

No writing assistance was utilized in the production of this manuscript.

\section{References}

1. Arabi AA. Routes to drug design via bioisosterism of carboxyl and sulfonamide groups. Future Med. Chem. 9(18), 2167-2180 (2017).

2. Ostrovskii VA, Trifonov RE, Popova EA. Medicinal chemistry of tetrazoles. Russ. Chem. Bull 61(4), 768-780 (2012).

3. Herr RJ. 5-Substituted-1H-tetrazoles as carboxylic acid isosteres: medicinal chemistry and synthetic methods. Bioorg. Med. Chem. 10(11), 3379-3394 (2002).

4. Kushwaha P, Fatima S, Upadhyay A et al. Synthesis, biological evaluation and molecular dynamic simulations of novel benzofuran-tetrazole derivatives as potential agents against Alzheimer's disease. Bioorg. Med. Chem. Lett. 29(1), 66-72 (2019).

5. Li Y, Pasunooti KK, Li RJ et al. Novel tetrazole-containing analogues of Itraconazole as potent antiangiogenic agents with reduced cytochrome P450 3A4 inhibition. J. Med. Chem. 61(24), 11158-11168 (2018).

6. Gao F, Xiao JQ, Huang G. Current scenario of tetrazole hybrids for antibacterial activity. Eur. J. Med. Chem. 184, e11174 (2019).

7. Zhang JY, Wang S, Ba YY, Xu Z. Tetrazole hybrids with potential anticancer activity. Eur. J. Med. Chem. 178, 341-351 (2019).

8. Wang SQ, Wang YF, Xu Z. Tetrazole hybrids and their antifungal activities. Eur. J. Med. Chem. 170, 225-234 (2019).

9. Gao C, Chang L, Xu Z et al. Recent advances of tetrazole derivatives as potential anti-tubercular and anti-malarial agents. Eur. J. Med. Chem. 163, 404-412 (2019).

10. Roh J, Karabanovich G, Vlckova H et al. Development of water-soluble 3,5-dinitrophenyl tetrazole and oxadiazole antitubercular agents. Bioorg. Med. Chem. 25(20), 5468-5476 (2017).

11. Zhan P, Li Z, Liu X, Clercq DE. Sulfanyltriazole/tetrazoles: a promising class of HIV-1 NNRTIs. Mini Rev. Med. Chem. 9(8), 1014-1023 (2009).

12. Gao F, Wang T, Xiao J, Huang G. Antibacterial activity study of 1,2,4-triazole derivatives. Eur. J. Med. Chem. 173, 274-281 (2019).

13. Gao F, Zhang X, Wang TF, Xiao JQ. Quinolone hybrids and their anti-cancer activities: an overview. Eur. J. Med. Chem. 165, 59-79 (2019).

14. Hu XL, Xu Z, Liu ML, Feng LS, Zang GD. Recent developments of coumarin hybrids as anti-fungal agents. Curr. Top. Med. Chem. 17, 3219-3231 (2017).

15. Kim TE, Lee H, Lim KS et al. Effects of HM30181, a p-glycoprotein inhibitor, on the pharmacokinetics and pharmacodynamics of loperamide in healthy volunteers. Br. J. Clin. Pharmacol. 78, 556-564 (2014).

16. World Health Organization. A global brief on hypertension: silent killer, global public health crisis. www.who.int/cardiovascular_diseases/publications/global_brief_hypertension/en/

17. Belz GG, Butzer R, Kober S, Mang C, Mutschler E. Time course and extent of angiotensin II antagonism after irbesartan, losartan, and valsartan in humans assessed by angiotensin II dose response and radioligand receptor assay. Clin. Pharmacol. Ther. 66, 367-373 (1999).

18. Feinstein D. Contrasting the neuroprotective and gliotoxic effects of PPAR $\gamma$ agonists. Drug Discov. Today 1(1), 29-34 (2004). 